\section{Response to Sierra-Delgado et al.}

To the Editor: In response to the remarks made by SierraDelgado and coauthors, ${ }^{1}$ we carefully reanalyzed our methods and re-examined our experimental set-up. ${ }^{2}$ Accordingly, we concluded that the setup, the results, and the conclusions of our experimental approach are correct.

In our original paper, "Similar Prevalence of Expanded CGG Repeat Lengths in the Fragile X Mental Retardation I Gene Among Infertile Women and Among Women With Proven Fertility: A Prospective Study," published in 2013, we determined the FMR1-CGG repeat length status using two different polymerase chain reaction (PCR) methods and a total of six different primers in a cohort of 620 women with well-established (in)fertility status. Although primer 3 is suitable for conventional PCR primer design, there are only two sets of thermodynamic parameters and three different salt correction formulas, ${ }^{3}$ which is not optimal for triplet repeat-primed PCR. Therefore, it is not surprising that Sierra-Delgado and coauthors did not find any primers matching those reported by us. In fact, some parameters are important for this type of reaction, such as additives (DMSO and betaine) in the reaction buffers.

Historically, FMR1-Fwd was named F and FMR1-Rev was C. They are the most used primers for determination of FMR1CGG repeats worldwide. ${ }^{4}$ In our work, the diagnostic accuracy of these primers has been independently and successfully validated using the following: (i) control DNA samples from the National Institute for Biological Standards and Control Reference Panel for Fragile X Syndrome (and used in each experiment as an internal control), (ii) control DNA samples from participations (with correct genotyping) at the European Quality Control Network (2007-2015), and (iii) commercially available PCR-based assays (AmplideX FMR1, Asuragen).

Concerning the presence of variants, based on the available data from the 1000 Genomes Project and the Exome Aggregation Consortium, variants are all rare to very rare, with minor allele frequencies (MAFs) of $0.007 \%$ up to $0.8 \%$ (K: rs781868385, MAF/MinorAlleleCount: $\mathrm{G}=0.0021 / 8$ (1000 Genomes); S: rs781928912, MAF/MinorAlleleCount: C = 0.00007/1 (ExAC); B: rs111485627, MAF/MinorAlleleCount: $\mathrm{T}=0.0040 / 15(1000$ Genomes) respectively. $\mathrm{T}=0.0079 / 121$ (ExAC); Figure 1). If we take the "worst" case (variant with $0.8 \% \mathrm{MAF}$ ), then the PCR would not have worked in 5 out of the 620 women studied. Because this would result in no PCR product, either it would show up as one allele only (when in reality two alleles of different sizes should have been amplified) or it would show up as no allele at all (when in reality two alleles of the same size are present). In any case, we would investigate such cases further because they might have high-range premutations missed by our assay using the Amplidex assay.

Although secondary structures (hairpins) of primers used and PCR efficiency were criticized, in our experiment we used the GC-Rich PCR system, which is suitable for FMR1 template because the system is composed of an enzyme blend of thermostable Taq DNA Polymerase and Tgo DNA Polymerase. The GC-RICH PCR Reaction buffer in combination with the separately included GC-RICH resolution solution allows very efficient amplification of difficult templates like GC-rich targets. During the reaction, higher annealing temperature $\left(64^{\circ} \mathrm{C}\right) \mathrm{can}$ also very efficiently remove secondary structures, if they exist.

We admit that the abbreviations "Fwd" and "Rev," which are routinely used in our laboratory, may be confused as "forward"

a

For primer FMR1-Rev:

TACACCCGCAGCGGGCC $\overline{G G G G G T T C G G C C T S A G T C A ̈ G C G C T C A G C T C C G T T T C G G T T T C D ~}$

ACTTCCGGKGGRGGCCGCCTCTGAGCGGGCGGCGGGCCGACGGCAACGCGGGCGGCG

K: rs781868385, MAF/MinorAlleleCount:G = 0.0021/8 (1,000 Genomes)

b

For primer FMR1-Fwd
GCCCACCTCTCGGGGGCGGGCTCCCGGCGCTAGCAGGGCTGAAGAGAAGATGGAGGAGCT
GSTGGTGGAATTCGGGCICCAATGGCGTTCTACAAGGTACTTGGCTCTAGGGCAGG
CCCCATCTTCGCCCTTCCTTCCCTCCCTTTCTTCTTGGTGTCGGCGGGAGGCAGGCCCG

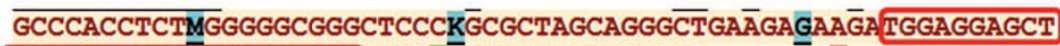

GSTGGTBGAAGTGCGGGGCTCCAAY G̈GCGCTTTCTACAAGGTACTTG GGCTCTAGGGCAGG

CCCCATCTT ENSE00001810087 [TCCCTCCCTTTTCTTCTTGGTGTCGGCGGGAGGCAGGCCCG

S: rs781928912, MAF/MinorAlleleCount:C $=0.00007 / 1$ (ExAC)

B: rs111485627, MAF/MinorAlleleCount: $T=0.0040 / 15$ (1,000 Genomes) resp. $T=0.0079 / 121$ (ExAC)

Figure 1 Details of FMR1 primers used (forward and reverse). 
and "reverse." However, we stress here that the denomination of a primer being "forward" or "reverse" in a noncoding region of a gene has no technical implication in experimental results.

We herewith refute the statement by Sierra-Delgado and coauthors ${ }^{2}$ and hope to convince the readership that the methods, results, and conclusions of our original paper ${ }^{1}$ are valid.

\section{DISCLOSURE}

The authors declare no conflict of interest.

Christian De Geyter, MD ${ }^{1,2}$, Nadira M'Rabet ${ }^{1}$, Julie De Geyter ${ }^{1}$, Hong Zhang, $\mathrm{PhD}^{1}$ and Karl Heinimann, $M D^{3}$

${ }^{1}$ Research Group on Gynecological Endocrinology, Department of Biomedicine, University Hospital, University of Basel, Basel, Switzerland; ${ }^{2}$ Clinic of Gynecological
Endocrinology and Reproductive Medicine, University Hospital, University of Basel, Basel, Switzerland; ${ }^{3}$ Research Group on Human Genetics, Department of Biomedicine, University of Basel, Basel, Switzerland. Correspondence: Christian De Geyter (Christian.DeGeyter@usb.ch)

\section{REFERENCES}

1. De Geyter C, M'Rabet N, De Geyter J, et al. Similar prevalence of expanded CGG repeat lengths in the fragile $X$ mental retardation I gene among infertile women and among women with proven fertility: a prospective study. Genet Med 2014;16:374-378.

2. Sierra-Delgado JA, Pérez VL, Vargas $\mathrm{Cl}$. Use of bioinformatic tools in primer validation. Genet Med 2016;18:853-854.

3. Rozen S, Skaletsky H. Primer3 on the WWW for general users and for biologist programmers. Methods Mol Biol 2000;132:365-386.

4. Fu Y-H, Kuhl DPA, Pizzuti A, et al. Variation of the CGG repeat at the fragile $X$ site results in genetic instability: resolution of the Sherman paradox. Cell 1991;67:1047-1058

Advance online publication 14 July 2016. doi:10.1038/gim.2016.79 